\title{
Molecular biology of cancer
}

\section{Last week's Nature conference in Boston suggests that the study of oncogenes will provide a route to general understanding as well as a powerful insight into normal cell biology.}

THE molecular biology of cancer may almost be synonymous with the molecular biology of the cell. That is one of the impressions formed by last week's conference on the molecular biology of cancer. What seems to be happening is that the exceptional circumstances of malignancy (or of transformed cells in culture) is throwing a clear light on many aspects of the normal functioning of normal cells. Who would have guessed, even a few years ago, that so much would now be known about the biochemistry of the previously shadowy materials known as growth factors, for example?

So is the molecular biology of cancer merely another way of tackling longstanding problems of an academic character? That is the naturally impatient question asked by physicians, epidemiologists and the managers of public health. Patients also ask questions on the subject, and for good reason.

The answer, which is in the negative, is nevertheless a little roundabout. That the best cure for cancer is avoidance is undeniable. The worry, for now and for some time to come, is whether avoidance is feasible on the scale and at the speed to which agencies such as the US National Cancer Institute are committing themselves. Everybody will be delighted if the objective of reducing cancer mortality in the United States by half by the year $\mathbf{2 0 0 0}$ can be attained. But even if the programme succeeds, there will still be more than 250,000 deaths a year from cancer in the United States. So the need for better treatment will still be as clamant, for which purpose only a better understanding of the manifestations of forms of the disease can provide.

\section{Speakers}

S. Broder (National Institutes of Health); J. Cairns (Harvard University); R. Gallo (National Institutes of Health); F. Gilbert (Mt Sinai School of Medicine); P. Greenwald (National Institutes of Health); J. Gutterman (M. D. Anderson Hospital, Texas); P. Leder (Harvard University); R. Peto (University of Oxford); M. Sporn (National Institutes of Health); G. Todaro (Oncogen Inc.); J. Uhr (University of Texas); A. Varshavsky (Massachusetts Institute of Technology); R. Weinberg (Massachusetts Institute of Technology); I. B. Weinstein (Columbia University); $\mathbf{R}$. White (University of Utah).
In this sense, the two approaches to the problem of cancer-avoidance and understanding - are complementary. But they are more closely linked even than that. The strategy that emphasizes avoidance (the best course) requires that people should change their ways, sometimes radically. Public authorities, which have a constitutional licence to fix tax rates and speed limits, are conspicuously successful at changing their electors' habits. Understanding, however, may in the long run accomplish what exhortation cannot. And the molecular biology of cancer is more likely to provide persuasive general understanding than some of the advertising campaigns manned for the years ahead.

John Maddox

\section{Transgenic mice inherit cancer}

MAKE your own oncogene, Dr Philip Leder's recipe for grafting the regulatory sequences of the mouse mammary tumour virus (MMTV) onto the coding sequences of the c-myc gene, was the biggest sensation last week.

The circumstances are clearer than the full significance. The c-myc gene appears to be responsible for human lymphomas when translocated from its normal position on chromosome 8 to somewhere among the immunoglobin genes on chromosomes 2, 14 and 22, presumably because the regulatory environment then allows it to be activated. But the myc gene is a curious structure, with a long (more than 100 base-pair) sequence upstream of the first of three exons, which is duly transcribed into RNA but which (together with a short stretch at the beginning of the second exon) plays no part in coding for the gene product. So why not equip the gene with indigenous control elements by replacing the upstream end by a known regulatory element?

Fusion genes on this pattern are made from the regulatory elements of MMTV and the myc gene from which various upstream regions have been removed. In fertilized mouse oocytes, the synthetic genes become part of the genetic constitution of transgenic mice. In adult females, the gene should be turned on during pregnancy, as MMTV is activated by hormonal influences.

The offspring of two of the transgenic mice produced in this series of experiments turn out to have inherited MMTVmyc fusion genes, most remarkably in the case of four daughters of a transgenic mouse provided with the bare minimum of c-myc-the coding parts of the second and third exons, the intervening sequence and the downstream tail of the gene.

The pathological effects of the synthetic oncogene are distinct from those of MMTV and of c-myc but result, rather, in a distinctive adenocarcinoma of the breast tissue appearing only after pregnancy (confirming the influence of the lactational hormones). Offspring have been salvaged from these pregnancies (transgenic females are bad mothers, perhaps not coincidentally) by fostering, and the females turn out to have inherited both the fusion genes and the characteristic pathology of their mothers.

The mere development of this mouse strain confirms current notions that c$m y c$ gene in the human genome is, in its normal position on chromosome 8 , repressed (by some unknown mechanism), but that translocation to some other site allows it to be expressed. In this respect, c-myc differs from the ras oncogene, the activated form of which is now known to produce a protein distinct from the normal variety.

Potentially, the new strain of transgenic mice is a valuable model for the study of gene regulation and of oncogenesis, or at least of that for which myc is responsible.

The view that myc is normally suppressed, which Leder describes as a speculation, is consistent with the association of human lymphoma (and its mouse equivalent) with translocation but also with comparisons of the structure of the long leader sequence ahead of the three myc exons in the genes from mouse and human beings. These have substantially diverged from each other in the course of evolution except for a short region of some 200 base-pairs, most graphically seen in heteroduplex structures made from a DNA strand from each species, which may be the site of repression.

Functionally, c-myc normally appears to be active only in the cell cycling of precursor white blood cells. In culture, the cell products appear 3-6 hours after the treatment of resting cells with platelet-derived growth factor (PGDF). Of the 